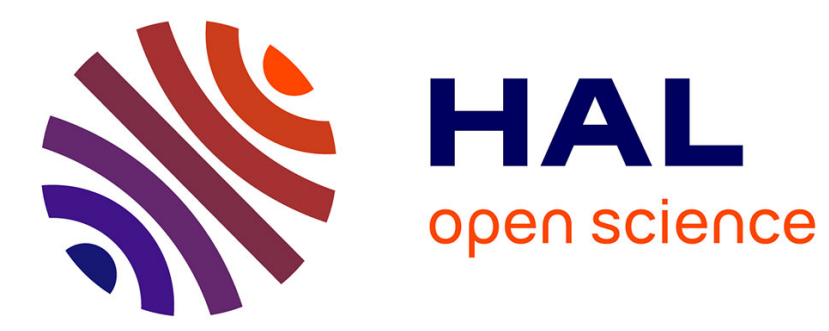

\title{
Solution to Sylvester equation associated to linear descriptor systems
}

\author{
Mohamed Darouach
}

\section{To cite this version:}

Mohamed Darouach. Solution to Sylvester equation associated to linear descriptor systems. Systems and Control Letters, 2006, 55 (10), pp.835-838. 10.1016/j.sysconle.2006.04.004 . hal-00105181

\section{HAL Id: hal-00105181 \\ https://hal.science/hal-00105181}

Submitted on 10 Oct 2006

HAL is a multi-disciplinary open access archive for the deposit and dissemination of scientific research documents, whether they are published or not. The documents may come from teaching and research institutions in France or abroad, or from public or private research centers.
L'archive ouverte pluridisciplinaire HAL, est destinée au dépôt et à la diffusion de documents scientifiques de niveau recherche, publiés ou non, émanant des établissements d'enseignement et de recherche français ou étrangers, des laboratoires publics ou privés. 


\title{
Solution to Sylvester equation associated to linear descriptor systems
}

\author{
M. Darouach \\ CRAN-CNRS (UMR 7039), Université Henri Poincaré, Nancy I \\ IUT de Longwy, 186 Rue de Lorraine \\ 54400 COSNES et ROMAIN \\ FRANCE \\ e-mail mohamed.darouach@iut-longwy.uhp-nancy.fr
}

\begin{abstract}
This paper presents the solution of the constrained Sylvester equation associated to linear descriptor systems. This problem has been recently studied in [1], where sufficient conditions for the existence of the solution are given. In the present paper, a simple and direct method is developed to solve this problem. This method shows that the conditions given in [1] are necessary and sufficient.
\end{abstract}

Keywords: Descriptor systems; Sylvester equation; Existence conditions; Observers; Regional pole placement

\section{Introduction}

Many problems in control and systems theory are solved by computing the solution of Sylvester equations. As it is well known, these equations have important applications in stability analysis, in observers design, in output regulation with internal stability, and in the eigenvalue assignment (see, e.g [1], [2], [3], [4]). Sylvester equations associated with descriptor systems (or generalized Sylvester equations) have received wide attention in the literature, see [1], [2], [3], [4]. Recently, sufficient conditions for the existence of the solution to these equations under a rank constraint have been given in [1]. The present paper considers the problem studied in [1]. A new approach to solve these equations is developed, necessary and sufficient conditions are presented.

Consider the linear time-invariant multivariable system described by

$$
\begin{aligned}
& E \dot{x}=A x+B u, \\
& y=C x,
\end{aligned}
$$

where $x \in \mathbb{R}^{n}$ is the state vector, $y \in \mathbb{R}^{p}$ the output vector, and $u \in \mathbb{R}^{m}$ the input vector. The matrices $E \in \mathbb{R}^{n \times n}, A \in \mathbb{R}^{n \times n}, B \in \mathbb{R}^{n \times m}$, and $C \in \mathbb{R}^{p \times n}$ are known constant matrices, with $\operatorname{rank}(E)=q<n, \operatorname{rank}(B)=m$ and $\operatorname{rank}(C)=p<q$.

Consider the Problem 1 studied in [1], which can be formulated as follows:

Let $\mathcal{D}$ be a region in the open left half complex plane, $\mathcal{D} \subseteq \mathbb{C}^{-}$, symmetric with respect to the real axis. The problem is to find matrices $T \in \mathbb{R}^{(q-p) \times n}, Z \in \mathbb{R}^{(q-p) \times n}$, and $H_{T} \in \mathbb{R}^{(q-p) \times(q-p)}$, such that 


$$
T A-H_{T} T E=-Z C
$$

with $\sigma\left(H_{T}\right) \subset \mathcal{D}$, under the rank constraint

$$
\operatorname{rank}\left[\begin{array}{c}
T E \\
L A \\
C
\end{array}\right]=n
$$

where $\sigma\left(H_{T}\right.$ is the spectrum of $H_{T}$ and $L \in \mathbb{R}^{(n-q) \times n}$ is any full row rank matrix satsfying $L E=0$.

The Sylvester equation (2) has a close relation with many problems in linear control theory of descriptor systems, such as the eigenstructure assignment [4], [5], and the state observer design. The observer design problem can be formulated as follows [1]:

For the descriptor system (1), consider a reduced-order observer of the form

$$
\begin{aligned}
& \dot{z}(t)=H_{T} z(t)+T B u(t)-Z y(t), \\
& \hat{x}(t)=S z(t)+\bar{N} \bar{y}+N y(t),
\end{aligned}
$$

where $z \in \mathbb{R}^{(q-p)}$ is the state of the observer and $\bar{y} \in \mathbb{R}^{(n-q)}$ is a fictitious output. As shown in [1], if Problem 1 is solved for some matrices $T, Z$ and $H_{T}$ and if we compute the matrices $S, \bar{N}$ and $N$ such that $\left[\begin{array}{lll}S & \bar{N} & N\end{array}\right]\left[\begin{array}{c}T E \\ L A \\ C\end{array}\right]=I$, then, for observer (4) we have:

i) $\lim _{t \rightarrow \infty}(z(t)-T E x(t))=0$,

ii) for $\bar{y}(t)=-L B u(t)$, the estimated state $\hat{x}(t)$ satisfies $\lim _{t \rightarrow \infty}(x(t)-\hat{x}(t))=0$.

Remark 1 Notice that for $L=0$, Problem 1 is reduced to finding matrices $T \in \mathbb{R}^{(n-p) \times n}$, $Z \in \mathbb{R}^{(n-p) \times n}$, and $H_{T} \in \mathbb{R}^{(n-p) \times(n-p)}$, such that

$$
T A-H_{T} T E=-Z C
$$

with $\sigma\left(H_{T}\right) \subset \mathcal{D}$, under the rank constraint

$$
\operatorname{rank}\left[\begin{array}{c}
T E \\
C
\end{array}\right]=n
$$

These conditions are those required for the observer design with order $(n-p)$, see [5].

\section{Main results}

In this section we will present a new and simple solution to Problem 1. Necessary and sufficient conditions to solve this problem are also given.

Since matrix $E \in \mathbb{R}^{n \times n}$ is singular and $\operatorname{rank}(E)=q<n$, there exist two nonsingular matrices $P$ and $Q$ of appropriate dimensions such that

$E_{c}=P E Q=\left[\begin{array}{cc}I_{q} & 0 \\ 0 & 0\end{array}\right], A_{c}=P A Q=\left[\begin{array}{ll}A_{11} & A_{12} \\ A_{21} & A_{22}\end{array}\right], C Q=\left[\begin{array}{ll}C_{1} & C_{2}\end{array}\right], T P^{-1}=\left[\begin{array}{ll}T_{1} & T_{2}\end{array}\right]$,

and $L P^{-1}=\left[\begin{array}{ll}L_{1} & L_{2}\end{array}\right]$.

According to this partitioning, equation $L E=0$ becomes $\left[\begin{array}{ll}L_{1} & L_{2}\end{array}\right] E_{c}=0$, which leads to $L_{1}=0$ and $L_{2} \in \mathbb{R}^{(n-q) \times(n-q)}$ is an arbitrary nonsingular matrix, since $L$ is of full row rank. 
Therefore, equation (3) can be written as

$$
\operatorname{rank}\left[\begin{array}{c}
T E \\
L A \\
C
\end{array}\right]=\operatorname{rank}\left[\begin{array}{c}
T P^{-1} E_{c} \\
L P^{-1} A_{c} \\
C Q
\end{array}\right]=\operatorname{rank}\left[\begin{array}{cc}
T_{1} & 0 \\
L_{2} A_{21} & L_{2} A_{22} \\
C_{1} & C_{2}
\end{array}\right]=n,
$$

or equivalently

$$
\operatorname{rank}\left[\begin{array}{c}
T E \\
L A \\
C
\end{array}\right]=\operatorname{rank}\left[\begin{array}{cc}
T_{1} & 0 \\
A_{21} & A_{22} \\
C_{1} & C_{2}
\end{array}\right]=n
$$

since $L_{2}$ is a nonsingular matrix.

Now equation (2) can be written as

$$
T P^{-1} A_{c}-H_{T} T P^{-1} E_{c}=-Z C Q,
$$

then using the above results we obtain

$$
\begin{aligned}
& T_{1} A_{11}-H_{T} T_{1}=\mathbf{J C}_{1}, \\
& T_{1} A_{12}=\mathbf{J C}_{2},
\end{aligned}
$$

with $\sigma\left(H_{T}\right) \subset \mathcal{D}$, and where $\mathbf{J}=-\left[\begin{array}{ll}T_{2} & Z\end{array}\right], \mathbf{C}_{1}=\left[\begin{array}{c}A_{21} \\ C_{1}\end{array}\right]$, and $\mathbf{C}_{2}=\left[\begin{array}{c}A_{22} \\ C_{2}\end{array}\right]$.

Define the following matrices $\mathbf{C}=\left[\begin{array}{ll}\mathbf{C}_{1} & \mathbf{C}_{2}\end{array}\right]$ and $\mathbf{T}=T E Q$, then equation (10) becomes

$$
\mathbf{T} A_{c}-H_{T} \mathbf{T}=\mathbf{J C}
$$

and equation (8) can be written as

$$
\operatorname{rank}\left[\begin{array}{l}
\mathbf{T} \\
\mathbf{C}
\end{array}\right]=n
$$

where $\mathbf{T}=T E Q=\left[\begin{array}{ll}T_{1} & 0\end{array}\right]$, which can also be written as

$$
\mathbf{T} F=0
$$

with $F=\left[\begin{array}{c}0 \\ I_{n-q}\end{array}\right]$.

Then Problem 1 reduces to finding matrices $\mathbf{T}, \mathbf{J}$, and $H_{T}$, with $\sigma\left(H_{T}\right) \subset \mathcal{D}$, such that (11) (13) are satisfied.

Remark 2 From the above results it is easy to see that condition rank $\left[\begin{array}{c}L A \\ C\end{array}\right]=n-q+p$ of theorem 1 of [1] is equivalent to $\mathbf{C}$ being a full row rank matrix.

Before proceeding, let us give the following definition which is useful for the sequel.

Definition 1. System (1) is $\mathcal{D}$-strongly detectable if and only if the following conditions are satisfied

$$
\begin{aligned}
& \text { 1) } \operatorname{rank}\left[\begin{array}{c}
A-\lambda E \\
C
\end{array}\right]=n, \forall \lambda \in \mathbb{C}, \lambda \notin \mathcal{D} \text {, } \\
& \text { 2) } \operatorname{rank}\left[\begin{array}{c}
E \\
L A \\
C
\end{array}\right]=n
\end{aligned}
$$


For the standard systems, where $\mathrm{E}=\mathrm{I}$, Definition 1 becomes.

Definition 2. Let $A \in \mathbb{R}^{n \times n}$ and $C \in \mathbb{R}^{p \times n}$ be constant matrices, then the pair $(C, A)$ is said to be $\mathcal{D}$-detectable if and only if

$$
\operatorname{rank}\left[\begin{array}{c}
\lambda I-A \\
C
\end{array}\right]=n, \forall \lambda \in \mathbb{C}, \lambda \notin \mathcal{D} .
$$

Before we give our main results, we establish the following lemma.

Lemma 1. The following statements are equivalent

1) System (1) is $\mathcal{D}$-strongly detectable,

2) $\operatorname{rank}\left[\begin{array}{cc}\lambda I-A_{c} & F \\ \mathbf{C} & 0\end{array}\right]=2 n-q, \forall \lambda \in \mathbb{C}, \lambda \notin \mathcal{D}$, and $\operatorname{rank}\left[\begin{array}{c}A_{22} \\ C_{2}\end{array}\right]=n-q$.

Proof. By using the above transformations we obtain:

$$
\operatorname{rank}\left[\begin{array}{c}
A-\lambda E \\
C
\end{array}\right]=\operatorname{rank}\left[\begin{array}{c}
P(A-\lambda E) Q \\
C Q
\end{array}\right]=\operatorname{rank}\left[\begin{array}{cc}
\lambda I-A_{11} & -A_{12} \\
-A_{21} & -A_{22} \\
C_{1} & C_{2}
\end{array}\right],
$$

and

$$
\operatorname{rank}\left[\begin{array}{c}
E \\
L A \\
C
\end{array}\right]=\operatorname{rank}\left[\begin{array}{c}
P E Q \\
L P^{-1} P A Q \\
C Q
\end{array}\right]=\operatorname{rank}\left[\begin{array}{cc}
I_{q} & 0 \\
L_{2} A_{21} & L_{2} A_{22} \\
C_{1} & C_{2}
\end{array}\right]=q+\operatorname{rank}\left[\begin{array}{c}
A_{22} \\
C_{2}
\end{array}\right]
$$

which prove the lemma.

The necessary and sufficient conditions for the existence of the solution to Problem 1 are then given by the following theorem.

Theorem 1. Under the assumption that matrix $\mathbf{C}$ is of full row rank, or rank $\mathbf{C}=n-q+p$, the necessary and sufficient condition for the existence of the solution to Problem 1 is that system (1) must be $\mathcal{D}$-strongly detectable.

Proof. Under condition (12), there exists a matrix $\mathbf{R}$, such that $\mathbf{T}=\mathbf{R}-K \mathbf{C}$ and $\operatorname{rank}\left[\begin{array}{l}\mathbf{T} \\ \mathbf{C}\end{array}\right]=$ $\operatorname{rank}\left[\begin{array}{cc}I & -K \\ 0 & I\end{array}\right]\left[\begin{array}{l}\mathbf{R} \\ \mathbf{C}\end{array}\right]=n$, or $\operatorname{rank}\left[\begin{array}{l}\mathbf{R} \\ \mathbf{C}\end{array}\right]=n$. In this case (11) becomes equivalent to

$$
(\mathbf{R}-K \mathbf{C}) A_{c}-H_{T}(\mathbf{R}-K \mathbf{C})=\mathbf{J C}
$$

or

$$
H_{T} \mathbf{R}+K_{1} \mathbf{C}+K \mathbf{C} A_{c}=\mathbf{R} A_{c},
$$

where $K_{1}=\mathbf{J}-H_{T} K$. From equation (18) it is easy to see that the knowledge of matrices $\mathbf{R}, H_{T}$, $K_{1}$, and $K$ is necessary and sufficient to determine the matrices $T$ and $Z$ of the initial problem.

Now let $\left[\begin{array}{l}\mathbf{R} \\ \mathbf{C}\end{array}\right]^{-1}=\left[\begin{array}{ll}M_{1} & N_{1}\end{array}\right]$, then post multiplying the two sides of equation (18) by the nonsingular matrix $\left[\begin{array}{ll}M_{1} & N_{1}\end{array}\right]$ leads to

$$
H_{T}=\mathbf{R} A_{c} M_{1}-K \mathbf{C} A_{c} M_{1}
$$

and

$$
K_{1}=\mathbf{R} A_{c} N_{1}-K \mathbf{C} A_{c} N_{1} .
$$


On the other hand, equation (13) can be written as $(\mathbf{R}-K \mathbf{C}) F=0$, or

$$
K \mathbf{C} F=\mathbf{R} F .
$$

The necessary and sufficient condition for the existence of a solution to (21) is that rank $\mathbf{C} F=$ $\operatorname{rank}\left[\begin{array}{l}R F \\ C F\end{array}\right]=\operatorname{rank} F=n-q$, which is equivalent to $\left[\begin{array}{c}A_{22} \\ C_{2}\end{array}\right]$ being of full column rank. Under this condition, the solution to (21) is given by

$$
K=\mathbf{R} F(\mathbf{C} F)^{+}+Y\left(I-(\mathbf{C} F)(\mathbf{C} F)^{+}\right),
$$

where $A^{+}$is any generalised inverse of matrix $A$, satisfying $A A^{+} A=A$, and $Y$ is an arbitrary matrix of appropriate dimension. Then substituting this expression in (19) yields

$$
H_{T}=\Lambda-Y \Omega
$$

where $\Lambda=\mathbf{R} A_{c} M_{1}-\mathbf{R} F(\mathbf{C} F)^{+} \mathbf{C} A_{c} M_{1}$ and $\Omega=\left(I-(\mathbf{C} F)(\mathbf{C} F)^{+}\right) \mathbf{C} A_{c} M_{1}$.

From $(23), \sigma\left(H_{T}\right) \subset \mathcal{D}$ if and only if the pair $(\Omega, \Lambda)$ is $\mathcal{D}$-detectable, or equivalently rank $\left[\begin{array}{c}\lambda I-\Lambda \\ \Omega\end{array}\right]=$ $q-p, \forall \lambda \in \mathbb{C}, \lambda \notin \mathcal{D}$.

Now, define the following full column matrices $S_{1}=\left[\begin{array}{cc}\mathbf{R} & 0 \\ \mathbf{C} & 0 \\ 0 & I\end{array}\right], S_{2}=\left[\begin{array}{cc}I & \mathbf{R} F(\mathbf{C} F)^{+} \\ 0 & I-(\mathbf{C} F)(\mathbf{C} F)^{+} \\ 0 & \mathbf{C} F)^{+}\end{array}\right]$, and the nonsingular matrix $S_{3}=\left[\begin{array}{ccc}M_{1} & N_{1} & 0 \\ 0 & 0 & I\end{array}\right]$, then we have

$$
\begin{aligned}
\operatorname{rank}\left[\begin{array}{cc}
\lambda I-A_{c} & F \\
\mathbf{C} & 0
\end{array}\right] & =\operatorname{rank} S_{1}\left[\begin{array}{cc}
\lambda I-A_{c} & F \\
\mathbf{C} & 0
\end{array}\right] S_{3} \\
& =n-q+p+\operatorname{rank} S_{2}\left[\begin{array}{cc}
\lambda I-\mathbf{R} A_{c} M_{1} & \mathbf{R} F \\
-\mathbf{C} A_{c} M_{1} & \mathbf{C} F
\end{array}\right] \\
& =2 n-2 q+p+\operatorname{rank}\left[\begin{array}{c}
\lambda I-\Lambda \\
\Omega
\end{array}\right]=2 n-q
\end{aligned}
$$

if and only if the pair $(\Omega, \Lambda)$ is $\mathcal{D}$-detectable, and using lemma 1 , we obtain the result of the theorem.

From the above results we can give the algorithm to solve Problem 1.

\subsection{Algorithm}

Let the conditions of theorem 1 be satisfied, then the following method computes the solution of Problem 1.

Step1: Compute matrices $P$ and $Q$ such that $P E Q=\left[\begin{array}{ll}I & 0 \\ 0 & 0\end{array}\right]$, then compute $A_{c}=P A Q=$ $\left[\begin{array}{ll}A_{11} & A_{12} \\ A_{21} & A_{22}\end{array}\right]$ and $C Q=\left[\begin{array}{ll}C_{1} & C_{2}\end{array}\right]$

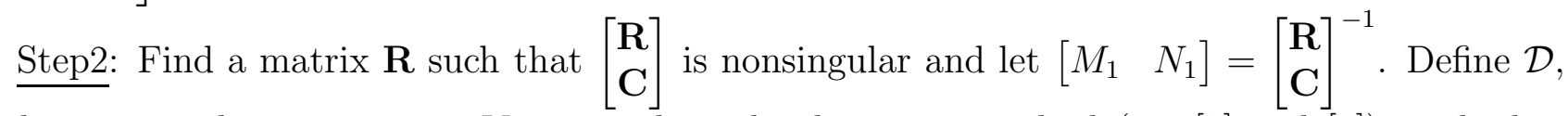
and compute the gain matrix $Y$, using the pole placement method (see [7] and [8]), such that $H_{T}=\Lambda-Y \Omega$, and $\sigma\left(H_{T}\right) \subset \mathcal{D}$, then compute $K_{1}$ and $K$ from (20) and (22). 
Step3: Compute $\mathbf{J}=K_{1}+H_{T} K, \mathbf{T}=\mathbf{R}-K \mathbf{C}, T_{1}=\mathbf{T}\left[\begin{array}{c}I_{q} \\ 0\end{array}\right], T_{2}=-\mathbf{J}\left[\begin{array}{c}I_{n-q} \\ 0\end{array}\right]$, then deduce $Z=-\mathbf{J}\left[\begin{array}{c}0 \\ I_{p}\end{array}\right]$ and $T=\left[\begin{array}{ll}T_{1} & T_{2}\end{array}\right] P$.

We can make the following remarks.

Remark 3 Contrary to the algorithm presented in [1], where many transformations are used in addition to the resolution of a Sylvester equation, in our paper the problem is reduced to a pole placement one which can be solved by the existing robust methods.

Remark 4 When $\operatorname{rank}(\mathbf{C})=n-q+p-d$, for some integer $0<d \leqslant n-q$, we can apply the above approach to this case, if and only if system (1) is $\mathcal{D}$-detectable.

Remark $\mathbf{5}$ In the above result, we have introduced a full row rank matrix $\mathbf{R}$ such that $\left[\begin{array}{l}\mathbf{R} \\ \mathbf{C}\end{array}\right]$ is nonsingular. The choice of this matrix is non unique, one can see that the results are independent of this choice. In fact, let $\overline{\mathbf{R}}$ be another full row rank matrix such that $\left[\begin{array}{l}\overline{\mathbf{R}} \\ \mathbf{C}\end{array}\right]$ is nonsingular, then there exists a regular matrix $\theta$ such that $\theta \overline{\mathbf{R}}=\mathbf{R}$. By premultiplying (17) by $\theta$ we obtain $(\overline{\mathbf{R}}-\bar{K} \mathbf{C}) A_{c}-\bar{H}_{T}(\overline{\mathbf{R}}-\bar{K} \mathbf{C})=\overline{\mathbf{J}} \mathbf{C}$, where $\overline{\mathbf{J}}=\theta \mathbf{J}, \bar{H}_{T}=\theta H_{T} \theta^{-1}$, and $\sigma\left(\bar{H}_{T}\right)=\sigma\left(H_{T}\right)$, which shows that the solution given above is independent of the choice of matrix $\mathbf{R}$.

\subsection{Regional pole placement in LMI regions}

This section discusses the solution of Problem 1 by regional pole placement in LMI regions of the complex plane. We shall use the same notations as in [1] and define the LMI type region $\mathcal{D} \subseteq \mathbb{C}^{-}$by the existence of two matrices $\Delta=\Delta^{T} \in \mathbb{R}^{(q-p) \times(q-p)}$ and $\Gamma \in \mathbb{R}^{(q-p) \times(q-p)}$ such that $\mathcal{D}=\left\{\lambda \in \mathbb{C}: f_{\mathcal{D}}(\lambda)=\Delta+\lambda \Gamma+\bar{\lambda} \Gamma^{T}<0\right\}$, then we propose the following result.

Theorem 2. Let $\mathcal{D} \subseteq \mathbb{C}^{-}$be an LMI type region, and assume that system (1) is $\mathcal{D}$-strongly detectable, then there exists a solution to Problem 1, with $\sigma\left(H_{T}\right) \subset \mathcal{D}$ if and only if there exist a symmetric positive definite matrix $X \in \mathbb{R}^{(q-p) \times(q-p)}$ and a matrix $\Psi \in \mathbb{R}^{(q-p) \times(q-p)}$ such that

$$
\Delta \otimes X+\Gamma \otimes(X \Lambda)-\Gamma \otimes(\Psi \Omega)+\Gamma^{T} \otimes(X \Lambda)^{T}-\Gamma^{T} \otimes(\Psi \Omega)^{T}<0,
$$

where $\Lambda=\mathbf{R} A_{c} M_{1}-\mathbf{R} F(\mathbf{C} F)^{+} \mathbf{C} A_{c} M_{1}, \Omega=\left(I-(\mathbf{C} F)(\mathbf{C} F)^{+}\right) \mathbf{C} A_{c} M_{1}$, and $\otimes$ is the Kronecker's product. In this case $H_{T}=\Lambda-Y \Omega$, with $Y=X^{-1} \Psi$.

Proof. The proof is direct, in fact from [6], $\sigma\left(H_{T}\right) \subset \mathcal{D}$ if and only if there exists a symmetric positive definite matrix $X \in \mathbb{R}^{(q-p) \times(q-p)}$ such that

$$
\Delta \otimes X+\Gamma \otimes\left(X H_{T}\right)+\Gamma^{T} \otimes\left(X H_{T}\right)^{T}<0 .
$$

From the above result we have $H_{T}=\Lambda-Y \Omega$, and by substituting this value in (25), and by putting $\Psi=X Y$ we obtain the desired result.

\section{Conclusion}

In this paper, we have presented a simple method to solve the generalized Sylvester equation associated to descriptor systems observers and control design. This problem was considered in [1], where only sufficient conditions were given. We have also given the necessary and sufficient conditions for the existence of the solution. Solution by LMI regional pole placement was also presented. 


\section{References}

[1] E.B. Castelan, V.G da Silva, On the solution of a Sylvester equation appearing in descriptor systems control theory, Systems Control Lett. 54 (2005) 109-117.

[2] M.Darouach, M.Boutayeb, Design of observers for descriptor systems, IEEE Trans, Automat. Control 40 (7) (1995) 1323-1327.

[3] M.Darouach, M.Zasadzinski, M.Hayar, Reduced-order observers design for descriptor systems with unknown inputs, IEEE Trans, Automat. Control 41 (1996) 1068-1072.

[4] L.Fletcher, Eigenstructure assignment by output feedback in descriptor systems, IEEE Proc. 135 (4) (1988) 302-308.

[5] A.Varga, On stabilization methods of descriptor systems, Systems Control Lett. 24 (2) (1995) 133-138.

[6] M.Chilali, P.Gahinet, $H_{\infty}$ Design with pole placement constraint: an LMI approach, IEEE Trans, Automat. Control 41 (3) (1996) 358-366.

[7] R.Byers, S.G.Nash, Approaches to robust pole assignment, Int.J.Control. 49 (1) (1989) 97117.

[8] A.L.Tits, Y.Yang, Globally convergent algorithms for robust pole assignment by state feedback, IEEE Trans, Automat. Control 41 (1996) 1432-1452. 